“C 2019 IEEE. Personal use of this material is permitted. Permission from IEEE must be obtained for all other uses, in any current or future media, including reprinting/republishing this material for advertising or promotional purposes, creating new collective works, for resale or redistribution to servers or lists, or reuse of any copyrighted component of this work in other works." 


\title{
Distributed Power Allocation Algorithm for General Authorised Access in Spectrum Access System
}

\author{
Ying $\mathrm{He}^{*}$, Beeshanga Abewardana Jayawickrama ${ }^{\dagger}$, Eryk Dutkiewicz* \\ ${ }^{*}$ University of Technology Sydney, Global Big data Technologies Centre, Australia \\ ${ }^{\dagger}$ Ericsson \\ E-mail: ying.he@uts.edu.au; beeshanga.jayawickrama@ericsson.com; eryk.dutkiewicz@uts.edu.au
}

\begin{abstract}
To meet the capacity needs of the next generation wireless communications, U.S. Federal Communications Commission has recently introduced Spectrum Access System. Spectrum is shared between three tiers - Incumbents, Priority Access Licensees (PAL) and General Authorised Access (GAA) Licensees. When the incumbents are absent, PAL and GAA share the spectrum under the constraint that GAA ensure the aggregate interference to $\mathrm{PAL}$ is no more than $\mathbf{- 8 0} \mathrm{dBm}$ within the PAL protection area. Currently GAA users are required to report their geolocations. However, geolocation is private information that GAA may not be willing to share. We propose a distributed GAA power allocation algorithm that does not require centralised coordination on sharing locations with other GAA users via SAS. We analytically proved the critical point of the interference along the PAL protection area to avoid calculating the interference on every points of the area. We proposed exclusion zone, transitional zone and open zone for GAA users to calculate the self-determined transmit power. Simulation results show that our method meets the interference requirement and achieve more than $90 \%$ of capacity approximation to the optimal centralised method, while completely masking the GAA locations.
\end{abstract}

Index Terms-Spectrum Access System, General Authorised Access, Priority Access Licensees, Aggregate Interference, Distributed Power Allocation

\section{INTRODUCTION}

Over the last few years, the capacity demands on cellular networks has increased drastically. To keep up with the growth of future cellular, more spectrum resources under $6 \mathrm{GHz}$ need to be allowed for use in cellular applications. To promote spectrum sharing as a potential solution the Federal Communications Commission (FCC) has proposed the Spectrum Access System (SAS) [1].

SAS proposed a three-tier sharing model. Incumbent users represent the highest tier in the framework and receive interference protection from Citizens Broadband Radio Service (CBRS) users. The CBRS consists of two tiers - Priority Access (PAL) and General Authorised Access (GAA). PAL/GAA licenses are issued by SAS for a finite census tract. PAL operations receive protection from GAA operations. In our previous work [2], we have studied the aggregate interference to the site location of the PAL users. Most existing works have also studied the aggregate interference to a pin-point [3] [4]. SAS further defined a PAL protection area outside of the PAL users. The aggregate interference is defined in Section 96.41(d)(1) [5] as that the co-channel aggregate interference shall not exceed $-80 \mathrm{dBm}$ in any direction within the PAL protection area. The existing methods on mitigating the interference to a pin-point cannot be directly applied to meet the requirement on the aggregate interference from all GAAs to the PAL protection area. Computation complexity for checking the interference from all GAAs on every single points along the boundary of the PAL protection area is very high.

To calculate the aggregate interference from all GAAs, most of the existing works require communication between GAA users or between GAA users and PAL users [6] [7] to share location information between them. This introduces high backhaul cost on inter-GAA and GAA-PAL communications and operators may be highly unwilling to disclose their site location information, as it is regarded as private information, as mentioned in item 327 [1]. Moreover, some existing works use exclusion zone [8] [9] and make binary decision on that the GAA users either transmit with the maximum power or cannot transmit. This saves computational cost but will leave the GAA users close to the PAL protection area with no chance to transmit. [10] shows the results between two category GAA users (high power and low power). However, the authors did not discuss about the interference to the PAL protection area.

We propose a method to find out the area where GAA can transmit with appropriate power level outside of the PAL protection area without the need to know the exact location of other GAA users. We analytically found and proved the critical point along the boundary of the PAL protection area. To the best of our knowledge, our method is the first distributed GAA power allocation that does not need the coordination with other GAA users via SAS and still have promising capacity compared to the optimal centralised method.

The rest of the paper is organised as follows: Section II introduces the system model. Section II presents the optimal centralised GAA resource allocation method that requires SAS to calculate the transmit power and allocate to each GAA. We propose a distributed GAA power allocation method in Section IV that GAA users can decide their transmit power according to their distance to the PAL protection area without the coordination with other GAAs via SAS. Simulation results and analysis are given in Section V. Conclusions are drawn in Section VI.

\section{SySTEM MODEL}

We consider the scenario where randomly distributed $N_{G}$ GAA users opportunistically access the spectrum in the pres- 
ence of $N_{P}$ PAL basestations. The maximum transmit power of the GAA users is $\hat{P}_{G}$. PAL and GAA users communicate with SAS (not with each other) using a control channel in the dedicated spectrum.

Considering its own transmission characteristics and the locations of basestations, the PAL users calculate the PAL protection zones. Protection zones are then reported to the SAS that makes this information publicly available for all GAAs. The GAA users are legally obliged to ensure that the aggregate interference is below a predetermined threshold $\lambda$ at all points within the protection zones.

All GAA users follow Listen Before Talk (LBT) protocol in any channel in $3550-3700 \mathrm{MHz}$ [1]. If a GAA user senses the received power is below a predetermined threshold $\mu$, it is free to start transmitting in that channel, at that instance, at that point in space. Maximum optimal transmit power is assigned by SAS in the current centralised approach, and it is selfdetermined in the proposed distributed approach. We assume the GAAs are either static or slowly moving. Our proposed approach calculates the transmit power of the GAA, such that the worst case aggregate interference in the PAL protection zone is below $\lambda$.

\section{Centralised GAA Resource Allocation}

The PAL protection zone is a finite area. Since the practical values of $\lambda$ is in the order of $-80 \mathrm{dBm}$, we can safely assume that active GAA transmitters are present only outside the PAL protection zone. In this scenario, interference can be managed as follows,

Proposition 1: If the aggregate interference from active GAAs at all points along the PAL protection zone boundary is below the threshold $\lambda$, the aggregate interference at all points inside the protection zone is less than $\lambda$.

Considering a generic pathloss model we write the following interference constraint,

$$
\sum_{j=1}^{N_{G}} K \frac{1}{d_{i j}^{\alpha}} P_{t}^{j} \leq \lambda ; \quad \forall i \in \Omega
$$

where $K$ and $\alpha$ are constants, $d_{i j}$ is the distance from $j$ th GAA to $i$ th point along the protection zone boundary, $P_{t}^{j}$ is the transmit power of the $j$ th GAA, and $\Omega$ is the set of all points along the PAL protection zone boundaries. As per Proposition 1, when (1) is satisfied, the GAAs meet the interference requirements.

In the current SAS framework, all GAA users should report their locations to SAS. Therefore the SAS is able to calculate the maximum power allocation for GAAs as follows,

$$
\begin{gathered}
\underset{P_{t}^{*}}{\arg \min }-\sum_{j=1}^{N_{G}} \log _{2}\left(1+P_{t}^{j}\right) \\
\text { subject to: } \quad \sum_{j=1}^{N_{G}} K \frac{1}{d_{i j}^{\alpha}} P_{t}^{j} \leq \lambda ; \quad \forall i \in \Omega
\end{gathered}
$$

Note that (2) is a convex problem that can be solved centrally at SAS. After SAS allocates power, a subset of $N_{G}$ GAAs

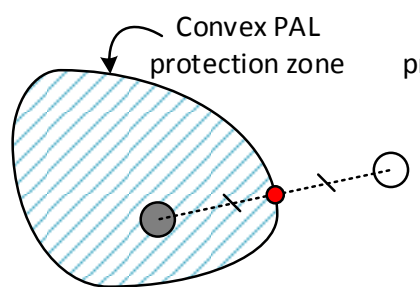

(a)

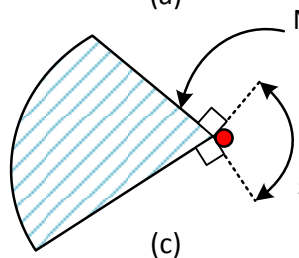

Not continuously differentiable

All GAAs in this area will have the same critical point

(c)

Fig. 1. Critical points of minimum distance to different possible PAL protection zones: (a) convex set (b) concave set (c) not continuously differentiable set. The mirror image of GAA could be either inside/outside the protection zone as shown.

transmit radio frames at any given instance following the LBT protocol as stated in Section II.

\section{Distributed GAA Power Allocation ALGORITHM}

In Section III we presented a centralised convex optimisation approach when all GAAs need to share their locations. However, GAA channel access pattern is expected to be sporadic in time, frequency and space domains. The channel access protocol will follow LBT. Therefore the GAA network topology (the set of active GAA nodes) changes rapidly from one radio frame to another. Hence having a distributed approach to power allocation will ensure the GAA network is able to rapidly adapt to such topology changes without the need to share or update their locations.

Consider the maximum transmit power $\hat{P}_{t}$ such that $P_{t}^{j} \leq$ $\hat{P}_{t} ; \forall 1 \leq j \leq N_{G}$. Let $\mu(<\lambda)$ be the LBT decision threshold such that $j$ th GAA begins transmission if the received aggregate power level is below $\mu$. Let $l_{j}$ be distance around which $j$ th GAA transmission is overheard by other GAA nodes. Hence we define,

$$
l_{j}=\left(\frac{K P_{t}^{j}}{\mu}\right)^{1 / \alpha} \quad \hat{l}=\left(\frac{K \hat{P}_{t}}{\mu}\right)^{1 / \alpha}
$$

where $\hat{l}$ is the corresponding $l_{j}$ value if $P_{t}^{j}=\hat{P}_{t}$. Hence, if the $j$ th GAA is already transmitting, all GAAs within a radius of $l_{j}$ will not begin transmission.

Let the PAL protection zone boundary $\Omega$ be a continuously differentiable set. Let $\omega_{j}(\in \Omega)$ be the set of critical points that gives the minimum distance from the $j$ th GAA to $\Omega$. There exists a many-to-one mapping between $\omega_{j}$ and the $j$ th GAA as shown in Fig. 1.

Proposition 2: Consider $j$ th and $k$ th unique GAAs that transmit at full power $\hat{P}_{t}$. Let the corresponding sets of critical 


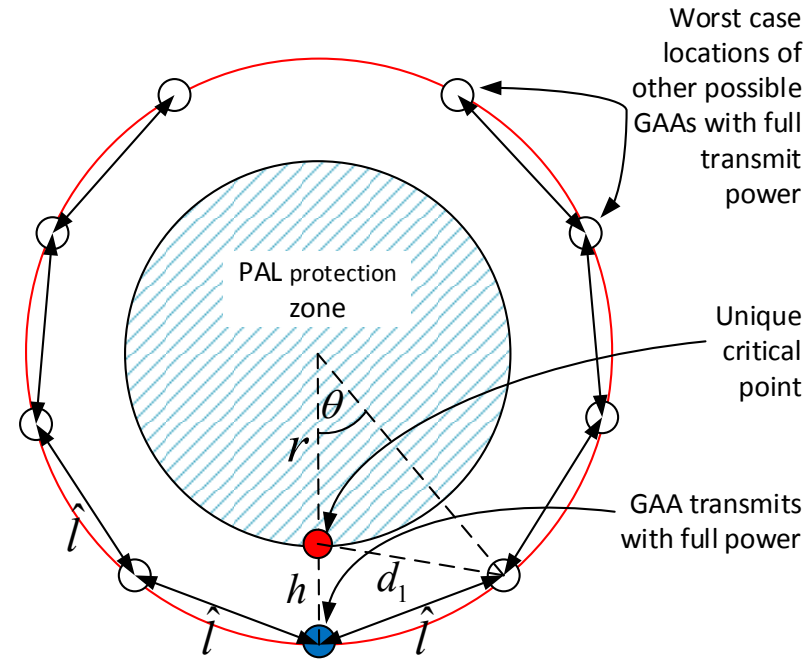

Fig. 2. Considering all GAAs that transmit at the maximum power $\hat{P}_{t}$, the worst case interference scenario is shown. Note that only the first tier of possible GAA locations is shown here.

points be $\omega_{j}$ and $\omega_{k}$, where the individual interference from each GAA reaches the same level. When $\Omega$ is a continuously differentiable set we have,

$$
\omega_{j} \cap \omega_{k} \equiv \emptyset
$$

Proof: We provide a proof by contradiction. Assume that there exists one element such that,

$$
\omega_{j} \cap \omega_{k}=\omega_{j k} \neq \emptyset
$$

where the power level of $j$ th and $k$ th GAAs reach $\mu$ (the proof is independent of the threshold value). Since $\Omega$ is continuously differentiable, the $j$ th GAA is $\hat{l}$ away from the $\omega_{j k}$ critical point, on the perpendicular line to the tangent of $\Omega$ at $\omega_{j k}$. Then the $k$ th GAA should be the mirror image of the $j$ th GAA, as shown in Fig. 1. The mirror image falls either within the protection zone or less than $\hat{l}$ away from some other point in $\Omega$. In both circumstances $k$ th GAA cannot transmit at $\hat{P}_{t}$.

Thus, we prove $\omega_{j} \cap \omega_{k} \equiv \emptyset$, which means the critical points of any two GAAs transmitting at $\hat{P}_{t}$ will be different.

Therefore we infer that, if $\Omega$ is a continuously differentiable set, the interference due to the $j$ th GAA all points in $\omega_{j}$ are the same, and the interference is strictly greater than all other points in $\Omega \backslash \omega_{j}$ and PAL protection zone. When $\Omega$ is convex $\omega_{j}$ has only one element.

Theorem 1: If all GAA users follow the same LBT protocol (with same $\mu$ ), and $\Omega$ is a continuously differentiable set, there exists a continuously differentiable set $\Omega$, on/outside which GAA users can transmit at the maximum allowed power $\hat{P}_{t}$ without having to coordinate with other GAAs via SAS.

Proof: Without loss of generality we do the proof when the PAL protection zone boundary $\Omega$ forms a circle (a convex set). Consider a convex set $\Omega$ that forms a larger circle with the same centre. Consider a GAA at one point on $\Omega$ that is transmitting at $\hat{P}_{t}$. In the worst case scenario, there could be GAAs transmitting at $\hat{P}_{t}$ on $\hat{\Omega}$ separated by at least $\hat{l}$ distance as shown in Fig. 2. We consider only the first tier of possible worst case GAA locations, the rest of the tiers are truncated.

Following the notations shown in Fig. 2 we write,

$$
\theta=\arccos \left(1-\frac{\hat{l}^{2}}{2(r+h)^{2}}\right)
$$

The distance from the critical point to the $n$th worst case location can be calculated as,

$$
d_{n}=\left(r^{2}+(r+h)^{2}-2 r(r+h) \cos (n \theta)\right)^{1 / 2}
$$

We define $H$ as follows,

$$
H=\left\{h \mid h \in \Re^{+}, \frac{\hat{l}}{2 \sin \left(\frac{2 \pi}{N}\right)}-r \leq h<\frac{\hat{l}}{2 \sin \left(\frac{2 \pi}{N+1}\right)}-r\right\}
$$

$\forall h \in H$, the aggregate interference $I$ at the critical point can be written as,

$$
I_{O}=\frac{K}{h^{\alpha}} \hat{P}_{t}+\sum_{n=1}^{N-1} \frac{K}{d_{n}^{\alpha}} \hat{P}_{t}
$$

where $N-1$ is the number of worst case GAA locations on S. (9) can be further converted into,

$$
I_{O}= \begin{cases}\frac{K}{h^{\alpha}} \hat{P}_{t}+2 \sum_{n=1}^{\eta} \frac{K}{d_{n}^{\alpha}} \hat{P}_{t} & N \text { is odd } \\ \frac{K}{h^{\alpha}} \hat{P}_{t}+2 \sum_{n=1}^{\eta} \frac{K}{d_{n}^{\alpha}} \hat{P}_{t}+\frac{K}{d_{N / 2}^{\alpha}} \hat{P}_{t} & N \text { is even }\end{cases}
$$

where $\eta$ follows,

$$
\eta=\left\lfloor\frac{\pi}{\theta}\right\rfloor= \begin{cases}\frac{N-1}{2} & N \text { is odd } \\ \frac{N}{2}-1 & N \text { is even }\end{cases}
$$

As $\eta$ in the summation is a function of $h$, and $\eta$ remains constant $\forall h \in H$. (10) is continuously differentiable in $h \in H$ and piecewise differentiable in $h \in \Re^{+}$. However when $r$ is sufficiently large, interference terms from the nodes that are furthest away from the critical point can be safely truncated. In this scenario we find the first order derivative as follows,

$$
\begin{aligned}
\frac{d}{d h} I_{O} & =-\alpha K \hat{P}_{t} \frac{1}{h^{\alpha+1}} \\
& + \begin{cases}-2 \alpha K \hat{P}_{t} \sum_{n=1}^{\eta} \frac{d_{n}^{\prime}}{d_{n}^{\alpha+1}} & N \text { is odd } \\
-2 \alpha K \hat{P}_{t} \sum_{n=1}^{\eta} \frac{d_{n}^{\prime}}{d_{n}^{\alpha+1}}-\alpha K \hat{P}_{t} \frac{d_{N / 2}^{\prime}}{d_{N / 2}^{\alpha+1}} & N \text { is even }\end{cases}
\end{aligned}
$$

where the first order derivative $d_{n}^{\prime}$ is as follows,

$$
d_{n}^{\prime}=\frac{(r+h)-r\left(\cos (n \theta)-(r+h) \sin (n \theta) n \theta^{\prime}\right)}{d_{n}}
$$




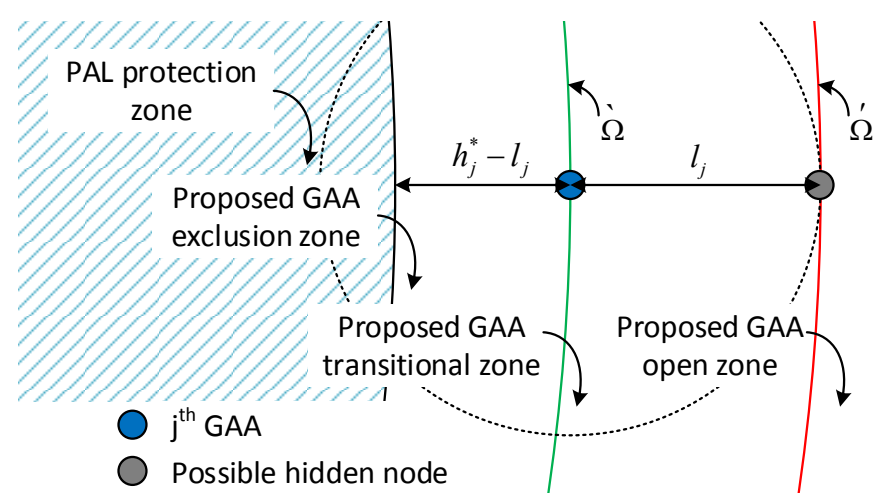

Fig. 3. Distributed GAA power allocation zones to meet the interference constraints within PAL protection zone.

where the first order derivative $\theta^{\prime}$ is as follows,

$$
\theta^{\prime}=-\frac{\hat{l} /(r+h)}{\sqrt{(r+h)^{2}-\hat{l}^{2} / 4}}
$$

Since $n \hat{l}<(r+h) \theta$ and $\theta<\pi, n \theta^{\prime}>-\frac{\pi}{\sqrt{(r+h)^{2}-\hat{l}^{2} / 4}}>-1$, when $r$ is sufficiently large. Therefore, we write the following for $\forall h \in H$,

$$
\frac{d}{d h} I_{O}<0
$$

Therefore, $I_{O}$ is a non-increasing function of $h, h \in H$. Thus the minimum possible value $h^{*}$ occurs when,

$$
\left.I_{O}\right|_{h=h^{*}}=\lambda
$$

Although it is difficult to find a closed form solution for $h^{*}$ in (16), using numerical techniques $h^{*}$ can be evaluated. Let $\Omega^{*}=\Omega \mid h=h^{*}$. The $h^{*}$ evaluated at all critical points in $\Omega$ will be the same. From Proposition 2, no two transmitters on $\Omega^{*}$ will have a common critical point which is a crucial condition for a distributed approach to be effective.

Therefore, there exists $\Omega^{*}$ outside which GAAs can follow LBT to transmit at $\hat{P}_{t}$ without the coordination of SAS, while meeting PAL protection zone interference requirements.

Consider a $j$ th GAA that is inside $\Omega$, and let the minimum distance between the $j$ th GAA and $\omega_{j}$ be $g$. We propose that the $j$ th GAA can transmit without coordinating with the other GAAs via SAS if and only if there exists a $P_{t}^{j}$ such that the worst case aggregate interference constraint is met at the critical points $\omega_{j}$ and $g<l_{j}$. If $g \geq l_{j}$, there could be hidden nodes on $\Omega$ that will not hear the $j$ th GAA. Therefore it is possible that the critical points $\omega_{k}$ of the $k$ th GAA on $\Omega$ to overlap with $\omega_{j}$. A distributed approach fails if (5) is violated.

Therefore similar to $\Omega$ introduced in Theorem 1 , there exists a continuously differentiable set $\grave{\Omega}$ inside which GAA users cannot transmit without coordinating with other GAAs via

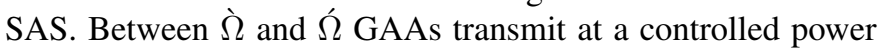
level. This leads to the zone layout shown in Fig. 3 .

In the transitional zone, $P_{t}^{j}$ can be calculated considering the worst case scenario where the full power GAAs on $\Omega$

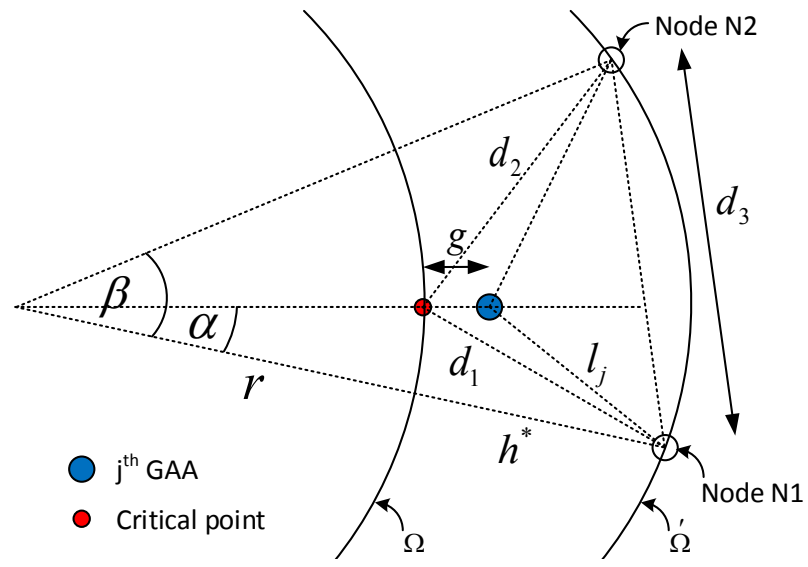

Fig. 4. Worst case scenario in the transitional zone.

Algorithm 1 Proposed distributed power allocation algorithm for $j$ th GAA outside the PAL protection zone

Input: SAS provides the PAL protection zone $\Omega$

1: Determine the minimum distance to $\Omega$ and the critical point.

2: Find $\Omega$ considering the worst case scenario, calculate $h^{*}$ from (16).

3: if Minimum distance to $\Omega \geq h^{*}$ then

4: $\quad$ GAA is in the open zone. Hence, $P_{t}^{j *}=\hat{P}_{t}$.

5: else if $\exists P_{t}^{j *}>0$ that satisfies (22) then

6: GAA is in the transitional zone, and the maximum 7: else transmit power is $P_{t}^{j *}$.$$
\text { 7: else }
$$$$
\text { 8: } \quad \text { GAA is in the exclusion zone, hence } P_{t}^{j *}=0 \text {. }
$$$$
\text { 9: end if }
$$

\section{Output: $P_{t}^{j *}$}

are closest to $\omega_{j}$. Without loss of generality when $\Omega$ and $\Omega$ are circles (convex sets), the worst case scenario is shown in Fig. 4. Both Node N1 and N2 transmit at the maximum power $\hat{P}_{t}$. With simple trigonometry followed by a few algebraic steps we find that,

$$
\begin{aligned}
& \alpha=\arccos \left(\frac{\left(r+h^{*}\right)^{2}+(r+g)^{2}-l_{j}^{2}}{2\left(r+h^{*}\right)(r+g)}\right) \\
& d_{1}=\left(r^{2}+\left(r+h^{*}\right)^{2}-2 r\left(r+h^{*}\right) \cos (\alpha)\right)^{1 / 2}
\end{aligned}
$$

The distance between two worst case GAA locations $d_{3}$ depends on the distance $g$. Consider only the two nearest possible locations of GAAs with transmit power $\hat{P}_{t}$ as shown in Fig. 4. The worst case interference occurs when N1 and N2 do not hear each other i.e. $d_{3} \geq \hat{l}$. If the GAA is closer to $\Omega$ than $\Omega$, the optimal $P_{t}^{j}$ will be sufficiently low that worst case scenario will be exactly as shown in Fig. 4. Otherwise $\mathrm{N} 2$ will be the mirror image of N1. Therefore, similar to (6) 


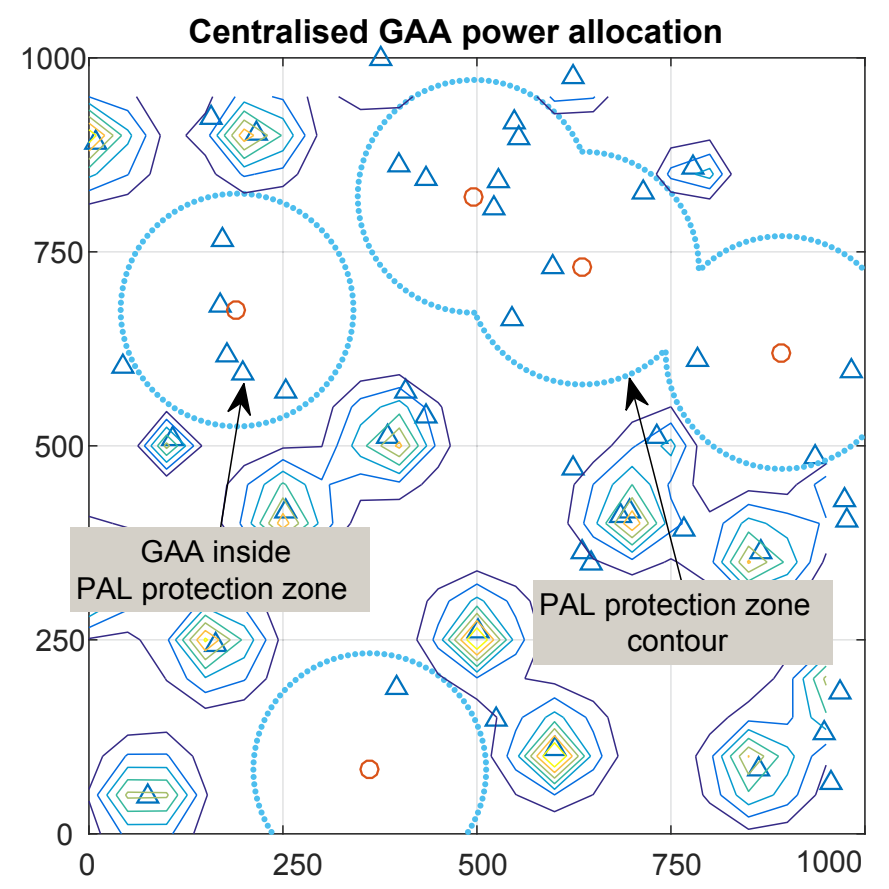

Fig. 5. Contour map of the aggregate GAA interference level for the centralised GAA power allocation approach (one iteration shown).

we write the following,

$$
\begin{aligned}
& \beta=\arccos \left(1-\frac{\left(\max \left\{\hat{l}, 2\left(r+h^{*}\right) \sin (\alpha)\right\}\right)^{2}}{2\left(r+h^{*}\right)^{2}}\right) \\
& d_{2}=\left(r^{2}+\left(r+h^{*}\right)^{2}-2 r\left(r+h^{*}\right) \cos (\beta-\alpha)\right)^{1 / 2}
\end{aligned}
$$

Therefore in the transitional zone (i.e. $\alpha>0$ ), the aggregate interference at the critical point can be calculated as,

$$
I_{T}=K P_{t}^{j} \frac{1}{g^{\alpha}}+K \hat{P}_{t}\left(\frac{1}{d_{1}^{\alpha}}+\frac{1}{d_{2}^{\alpha}}\right)
$$

When the maximum possible transmit power is reached,

$$
K P_{t}^{j} \frac{1}{g^{\alpha}}+\left.K \hat{P}_{t}\left(\frac{1}{d_{1}^{\alpha}}+\frac{1}{d_{2}^{\alpha}}\right)\right|_{P_{t}^{j}=P_{t}^{j *}}=\lambda
$$

The value of $P_{t}^{j *}$ can be evaluated numerically if the $j$ th GAA is in the transitional zone i.e. $\alpha>0$.

The distributed approach to GAA power allocation is shown in Algorithm 1. We assume that PAL protection zones are publicly made available by SAS. Based on this information each GAA classifies itself as a user in one of the zones shown in Fig. 3. Then each GAA picks an appropriate power level independently.

\section{Simulation AND Analysis}

We consider an 1000x1000 square meters area with 5 PAL users and 80 GAA users randomly located within the area. We consider the radius of the PAL protection area is 150 meters and the minimum distance between GAA users is 10 meters.

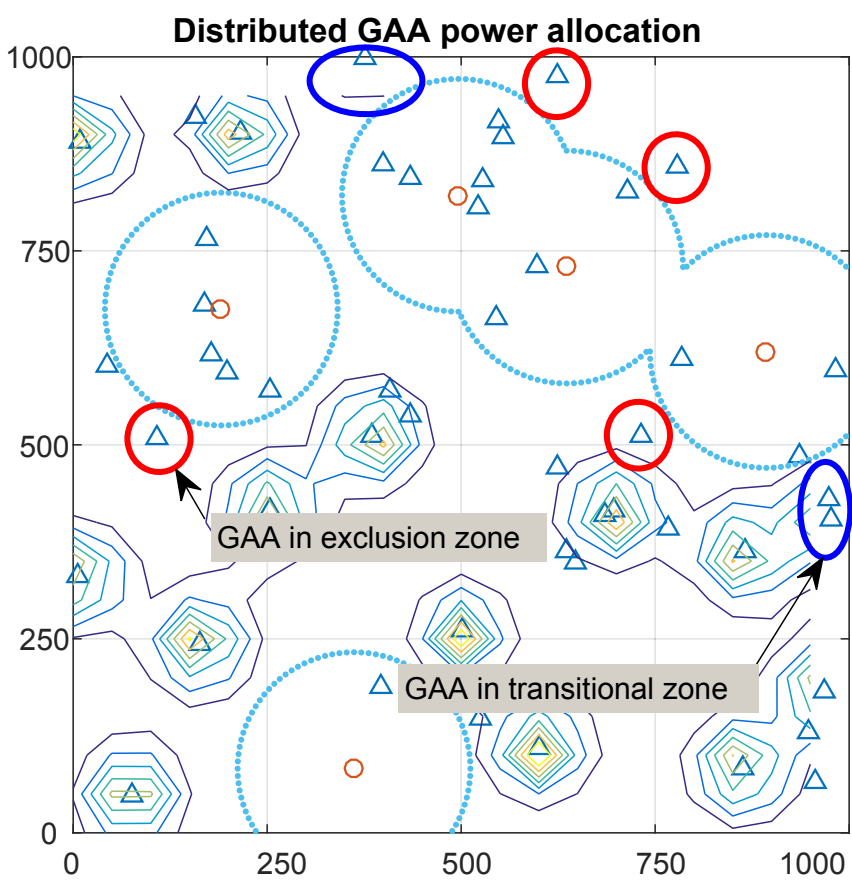

Fig. 6. Contour map of the aggregate GAA interference level for the proposed distributed GAA power allocation approach (one iteration shown).

We use the path loss model from [11], $P L(d B)=43.3 \log d+$ $11.5+20 \log f_{c}$ where the central frequency $f_{c}$ is $3.5 \mathrm{GHz}$. We consider the maximum transmit power as $24 \mathrm{dBm}$.

We first show a snapshot of the optimal result of the centralised power allocation approach in Fig. 5 . The $\triangle$ denotes a PAL user and $\circ$ denotes a GAA user. We show the scenario of overlapping PAL protection area too. The contour map around the GAA users shows the aggregate interference from all GAA users in the area. The most outer contour line denotes where the aggregate interference is $-80 \mathrm{dBm}$. The GAA users within the PAL protection area are not allowed to transmit. Similarly, we show a snapshot of our result of the proposed method in Fig. 6. Visually, we can tell that the aggregate interference meets the requirement as the most outer contour of the interference does not exceed the boundary of the PAL protection area. Moreover, we show the difference compared to Fig. 5. Following our method, some GAA users in the exclusion zone are not allowed to transmit, as illustrated in red circles. Some GAA users in the transitional zone are transmitting in a power that is lower than the maximum power level, as illustrated in blue circles. The rest other GAAs in the open zone are allowed to transmit with the maximum power level.

We further show the simulation results of the comparison of our proposed sub-optimal distributed method and the optimal centralised method in terms of the GAA network average downlink capacity in Fig. 7. The sub-optimal method achieves 90-90.33\% of the capacity with the optimal method. We can tell that for given number of the PAL users, the difference between the optimal and sub-optimal methods remains almost 


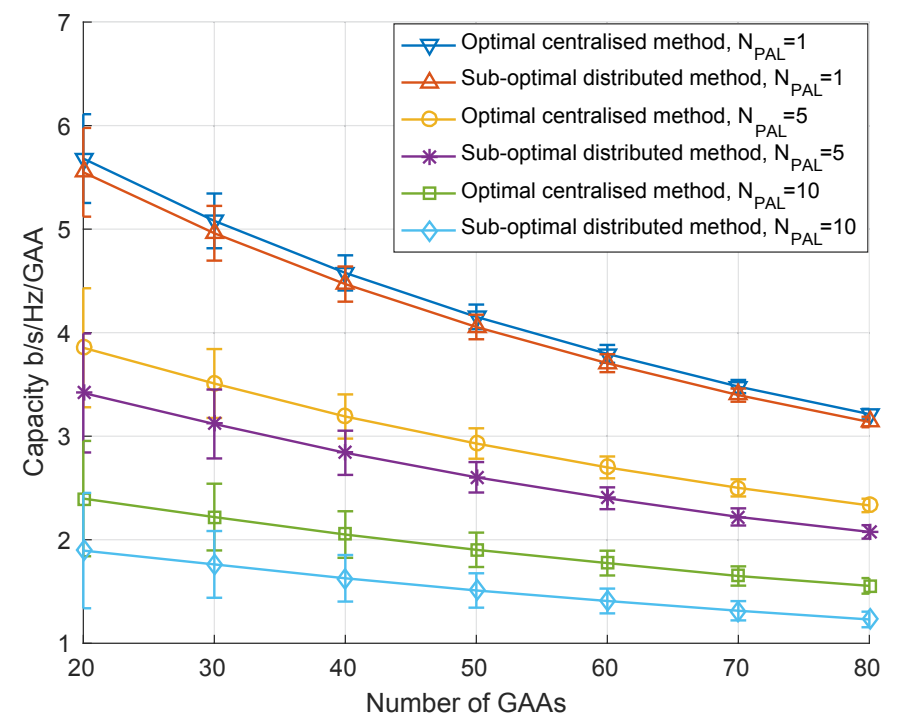

Fig. 7. Mean and the variance of average capacity per GAA.

stable. For given number of the GAA users, the difference increases with the increase of the number of the PAL users. This is due to that for a given area, the increase of the number of the PAL users leads to larger area of the exclusion zone and transitional zone, which results in more GAA users are allocated with zero or transmit power lower than the maximum power level.

In Fig. 8, we show the breakdown of the GAA network capacity in difference zones and the optimal method result in comparison. Compared to only considering GAAs to transmit in the open zone with maximum power, our approach boosts the capacity up to a better approximation (12.01-12.53\% in Fig. 8) to the optimal result by allowing the GAA users in the transitional zone to transmit.

\section{CONCLUSION}

In this paper, proposed a distributed power allocation algorithm for General Authorised Access users in Spectrum Access System. We defined and derived GAA exclusion zone, transitional zone and open zone to enable GAA users to decide the transmit power by themselves without coordinating with other GAA users via SAS centrally. This will substantially reduce the communication cost and delay between GAA users and between GAA and SAS. Simulation results show that our method met the aggregated interference requirement on the PAL protection area. The proposed distributed approach achieved over $90 \%$ of the capacity of the centralised approach, while completely masking the locations of GAAs.

\section{ACKNOWLEDGMENT}

This work has been supported by Intel's University Research Office. A US/EU patent (18175272.6-1214) has been filed for this work.

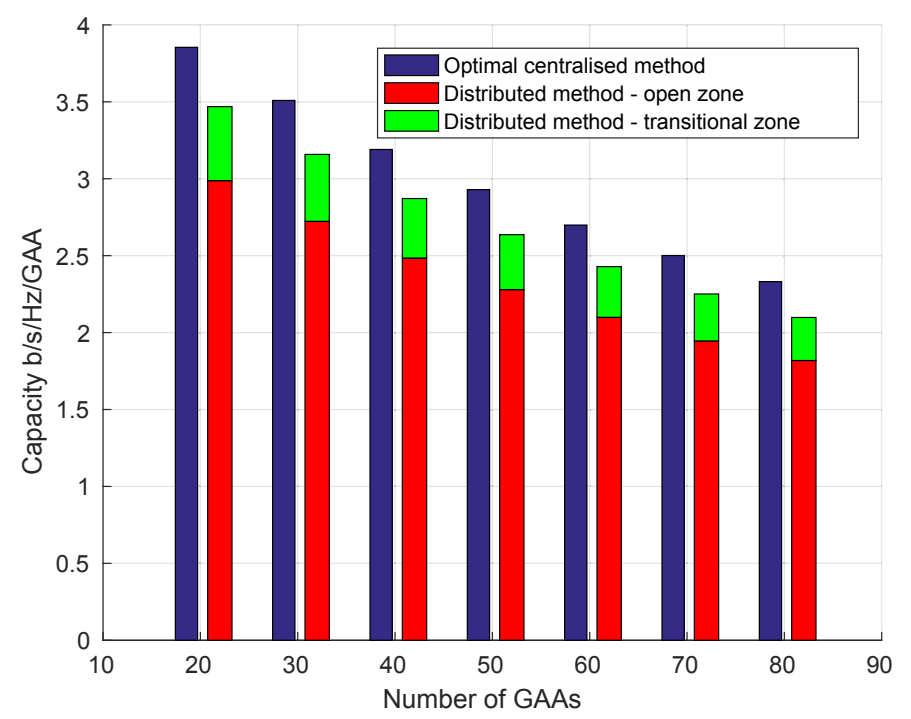

Fig. 8. Breakdown of average capacity in different zones.

\section{REFERENCES}

[1] Amendment of the Commission Rules with Regard to Commercial Operations in the $3550-3650 \mathrm{MHz}$ Band, Federal Communications Commission Std. GN Docket No. 12-354, Tech. Rep., Apr. 2015.

[2] Y. He, B. A. Jayawickrama, E. Dutkiewicz, S. Srikanteswara, and M. Mueck, "Priority access and general authorized access interference mitigation in the spectrum access system," IEEE Transactions on Vehicular Technology, vol. 67, no. 6, pp. 4969-4983, June 2018.

[3] T. Nadkar, V. Thumar, G. P. S. Tej, S. N. Merchant, and U. B. Desai, "Distributed power allocation for secondary users in a cognitive radio scenario," IEEE Transactions on Wireless Communications, vol. 11, no. 4, pp. 1576-1586, April 2012.

[4] Y. Xiao, G. Bi, and D. Niyato, "A simple distributed power control algorithm for cognitive radio networks," IEEE Transactions on Wireless Communications, vol. 10, no. 11, pp. 3594-3600, November 2011.

[5] Amendment of the Commission Rules with Regard to Commercial Operations in the 3550-3650 MHz Band, Federal Communications Commission Std. FCC 16-55 A1,GN Docket No. 12-354, Tech. Rep., May 2016.

[6] J. Denis, M. Pischella, and D. L. Ruyet, "Energy-efficiency-based resource allocation framework for cognitive radio networks with fbmc/ofdm," IEEE Transactions on Vehicular Technology, vol. 66, no. 6, pp. 4997-5013, June 2017.

[7] T. Zhang, W. Chen, Z. Han, and Z. Cao, "Hierarchic power allocation for spectrum sharing in ofdm-based cognitive radio networks," IEEE Transactions on Vehicular Technology, vol. 63, no. 8, pp. 4077-4091, Oct 2014.

[8] U. Tefek and T. J. Lim, "Interference management through exclusion zones in two-tier cognitive networks," IEEE Transactions on Wireless Communications, vol. 15, no. 3, pp. 2292-2302, March 2016.

[9] B. A. Jayawickrama, Y. He, E. Dutkiewicz, and M. D. Mueck, "Scalable spectrum access system for massive machine type communication," IEEE Network, vol. 32, no. 3, pp. 154-160, May 2018.

[10] R. Karaki and A. Mukherjee, "Coexistence of contention-based general authorized access networks in $3.5 \mathrm{ghz}$ cbrs band," in 2018 IEEE 87th Vehicular Technology Conference (VTC Spring), June 2018, pp. 1-6.

[11] Technical Specification Group Radio Access Network; Evolved Universal Terrestrial Radio Access (E-UTRA); Further advancements for E-UTRA physical layer aspects, 3GPP Std. Tech. Spec. 36.814 v9.0.0, Mar. 2010. 